\title{
Downregulation of matrix metalloproteinases contributes to the inhibition of cell migration and invasion in HepG2 cells by sodium valproate
}

\author{
XIA ZHAO ${ }^{1,2^{*}}$, WEIHUA YANG ${ }^{1 *}$, FENGYAN PEI $^{1}$, WANSHAN MA ${ }^{2}$ and YUNSHAN WANG ${ }^{1}$ \\ ${ }^{1}$ Medical Research \& Laboratory Diagnostic Center, Jinan Central Hospital, Shandong University, Jinan, Shandong 250013; \\ ${ }^{2}$ Department of Clinical Laboratory, Shandong Provincial Qianfoshan Hospital, Jinan, Shandong 250014, P.R. China
}

Received June 27, 2014; Accepted April 17, 2015

DOI: $10.3892 / \mathrm{ol} .2015 .3203$

\begin{abstract}
Histone deacetylation levels are closely associated with the genesis and development of tumors. However, the anti-tumor effect and mechanism of class I histone deacetylase inhibitor sodium valproate (VPA) on hepatocellular carcinoma cells has not been demonstrated clearly. In the present study, HepG2 hepatocellular carcinoma cells were exposed to VPA, and the effects of VPA on the proliferation, apoptosis, metastasis and invasion of the cells were evaluated. The MTT method and colony assays were used to detect cell proliferation, and cell migration and Transwell invasion were also evaluated. In addition, the protein expression levels of matrix metalloproteinase (MMP)-2 and MMP-9 were measured by flow cytometric analysis. The results indicated that VPA was able to inhibit proliferation and reverse the malignant phenotypes of hepatocellular carcinoma cells by inducing cell apoptosis. Furthermore, the colony formation and migration abilities of HepG2 cells were downregulated by VPA. Protein expression levels of MMP-2 and MMP-9 in HepG2 cells were also downregulated following VPA treatment, which contributed to suppression of the migration and invasion ability of HepG2 cells.
\end{abstract}

\section{Introduction}

Hepatocellular carcinoma is a polygenic and multifactorial malignant tumor, characterized by a complex mechanism, high level of malignancy, rapid progression, poor prognosis and high mortality rate (1). To date, surgery remains the

Correspondence to: Professor Yunshan Wang, Medical Research \& Laboratory Diagnostic Center, Jinan Central Hospital, Shandong University, 105 Jiefang Road, Jinan, Shandong 250013, P.R. China E-mail: sdjnwys@163.com

*Contributed equally

Key words: HepG2 cell, invasion, matrix metalloproteinase, migration, sodium valproate major therapeutic strategy for the treatment of hepatocellular carcinoma. Therefore, the development of novel preventive and therapeutic methods or drugs for hepatocellular carcinoma treatment is of significant interest. Modification of the N-terminal domains of the core histone is able to alter chromatin structures by modulating the affinity between the histone and DNA (2). Histone acetylation is regulated by histone acetyltransferase (HAT) and histone deacetylase (HDAC) (3). Previous studies have indicated that low levels of histone acetylation or high expression of HDACs are associated with the genesis and development of certain types of tumor; therefore HDAC inhibitors (HDACIs) $(4,5)$ were suggested as a potential tumor therapy method, which was verified by further research (6). However, certain HDACIs currently used in a clinical application are restricted due to their toxicity and short half-life (7). Sodium valproate (VPA), a widely used anticonvulsant drug, has been demonstrated to be a specific HDACI and is well known for its long half-life (8). However, the antineoplastic mechanism of VPA in hepatocellular carcinoma has not yet been fully elucidated.

The present study aimed to determine the effect of VPA in the hepatocellular carcinoma cell line, HepG2, in vitro. The protein expression levels of MMP-2 and MMP-9 were also detected to clarify the molecular mechanisms underlying of migration and invasion by regulating histone acetylation. Finally, the anti-hepatocellular carcinoma effect of VPA in vivo was investigated, using a mouse model of hepatocellular carcinoma.

\section{Materials and methods}

Cell culture and VPA treatment. HepG2 hepatocellular carcinoma cells (Cell Bank of Type Culture Collection of Chinese Academy of Sciences, Shanghai, China) were cultured in RPMI-1640 standard medium (Gibco Life Technologies, Carlsbad, CA, USA), supplemented with $10 \%$ fetal bovine serum, glutamine (Zhejiang Tianhang Biological Technology Co., Ltd., Huzhou, China) and antibiotics (50 IU penicillin and $50 \mu \mathrm{g} / \mathrm{ml}$ streptomycin; Sigma-Aldrich, St. Louis, MO, USA) in a humidified $5 \% \mathrm{CO}_{2}$ atmosphere at $37^{\circ} \mathrm{C}$. Exponentially growing HepG2 cells were incubated in six-well plates, at a concentration of $1 \times 10^{5}$ cells $/ \mathrm{ml}$. Following culture at $37^{\circ} \mathrm{C}$ with 
$5 \% \mathrm{CO}_{2}$ for $4 \mathrm{~h}, 10 \mu \mathrm{l}$ VPA (Sigma-Aldrich) was added at final concentrations of $0.75,1.5,2.0,3.0$ and $4.0 \mathrm{mmol} / 1$, respectively. The culture medium without VPA was used as control. There were three duplicate wells for each concentration gradient and cells were treated for 24, 48, 72 and 96 h. HepG2 cells were collected by centrifugation at each time-point.

Cell morphology and proliferation analysis. A total of $0.1 \mathrm{ml}$ exponentially growing HepG 2 cells $\left(5 \times 10^{4}\right.$ cells $\left./ \mathrm{ml}\right)$ were added to a 96 -well plate and cultured at $37^{\circ} \mathrm{C}$ with $5 \% \mathrm{CO}_{2}$ for $4 \mathrm{~h}$. Subsequently, VPA was added at $0.75,1.5,2.0,3.0$ and $4.0 \mathrm{mmol} / 1$ respectively and incubated in $5 \% \mathrm{CO}_{2}$ at $37^{\circ} \mathrm{C}$ for 24, 48, 72 and 96 h. Cell morphology was observed by Giemsa staining (Beijing Solarbio Science \& Technology Co., Ltd., Beijing, China) under a microscope (Leica Microsystems DMLB, Wetzlar, Germany). Cell proliferation was detected using the MTT method (9). Proliferation inhibition rate of cells $(\%)=($ number of control cells - number of VPA-treated cells)/number of control cells $\times 100 \%$.

Apoptosis assay. In accordance with the MTT assay results, HepG2 cells were treated with $0.75,1.5,2.0,3.0$ and $4.0 \mathrm{mmol} / 1$ VPA prior to detection of apoptosis. Cells were collected at 24 , 48, 72 and $96 \mathrm{~h}$, washed once with phosphate-buffered saline (PBS) and stained with Annexin V/propidium iodide (PI), according to the manufacturer's instructions of the Annexin V/PI Apoptosis Detection kit (Invitrogen Life Technologies, Carlsbad, CA, USA). Cells were subsequently analyzed by flow cytometry (FC 500, Beckman Coulter, Brea, CA, USA) and the results were analyzed using $\mathrm{EXPO}^{\mathrm{TM}} 32$ ADC software, version $1.1 \mathrm{C}$ (Beckman Coulter).

Colony formation assay. HepG2 cells were treated with VPA according to the aforementioned methods for 14 days. Subsequently, the culture solution was discarded and the cells were washed twice with PBS (pH 7.4; 0.1 mol/1; Sinopharm Chemical Reagent Co., Ltd, Shanghai, China). Cell colonies composed of $>50$ cells were counted under a microscope (Leica Microsystems DMLB) following Giemsa staining. The results were presented as the inhibition rate, according to the following formula: Colony formation inhibition rate $(\%)=($ control cell colony number - VPA treated cell colony number) / control cell colony number x $100 \%$.

Cell migration assay. HepG2 cells were incubated in six-well plates at a concentration of $2 \times 10^{5}$ cells/well. When $90 \%$ of the bottom of the wells were covered with a cell monolayer, cells were cultured in RPMI-1640 medium containing $1 \%$ fetal bovine serum, and the cells on two 20x5 mm areas were removed with cell scrapers (Corning Incorporated, Corning, NY, USA). The culture supernatant was replaced with $2 \mathrm{ml}$ fresh medium, containing $10 \%$ fetal bovine serum, and VPA $(0.75,1.5,2.0,3.0$ and $4.0 \mathrm{mmol} / \mathrm{l}$ ) was added. Following $24 \mathrm{~h}$ of culture, cells which had migrated into the scraped areas were counted under a microscope (Leica Microsystems DMLB). PBS was used as control. The results were presented as the inhibition rate, determined by: Cell migration inhibition rate $(\%)=$ (number of control cells migrated into scrapes - number of VPA-treated cells migrated into scrapes)/number of control cells migrated into scrapes $\mathrm{x} 100 \%$.
Cell invasion assay. The lower surfaces of the Transwell polycarbonate filters (Corning Incorporated) were coated with $0.1 \%$ gelatin (Beijing Solarbio Science \& Technology Co., Ltd.), and the upper surfaces were coated with $20 \mu$ l Matrigel (BD Pharmingen, San Diego, CA, USA). A total of $100 \mu 1$ exponentially growing HepG2 cells $\left(1 \times 10^{6}\right.$ cells $\left./ \mathrm{ml}\right)$ were added to the upper chambers of the Transwell apparatus with $0.75,1.5,2.0,3.0$ and $4.0 \mathrm{mmol} / \mathrm{l} \mathrm{VPA}$, while culture supernatant of NIH-3T3 cells (Cell Bank of Type Culture Collection of Chinese Academy of Sciences, Shanghai, China) was added to the lower chambers, and cultured for $24 \mathrm{~h}$ at $37^{\circ} \mathrm{C}$ with $5 \%$ $\mathrm{CO}_{2}$. HepG2 cells which had invaded to the lower surface were stained with Wright-Giemsa stain and counted under a microscope. The result was indicated as the inhibition rate, determined by: Cell invasion inhibition rate $(\%)=$ (number of control cells on the lower side - number of VPA-treated cells on the lower side)/number of control cells on the lower side $\mathrm{x} 100 \%$.

Protein expression of MMP-2 and MMP-9. Protein expression levels of MMP-2 and MMP-9 were measured by flow cytometry. Mouse monoclonal primary antibodies against MMP-2 (8B4; catalog no. SC-13595; Santa Cruz Biotechnology, Inc., Santa Cruz, CA, USA) and MMP-9 (2C3; catalog no. SC-21733; Santa Cruz Biotechnology, Inc.) were used. The primary antibodies were diluted (1:40) using PBS solution with $0.1 \%$ sodium azide. The secondary antibody was an FITC-conjugated polyclonal rabbit anti-mouse IgG (dilution, 1:40; H+L; catalog no. sc-358916; Santa Cruz Biotechnology, Inc.). Briefly, $5 \times 10^{6} \mathrm{HepG} 2$ cells were collected and washed following exposure to 1.5 or $3.0 \mathrm{mmol} / 1 \mathrm{VPA}$ for $48 \mathrm{~h}$. Cells were mixed with $1 \mathrm{ml}$ permeabilization buffer and incubated for $15 \mathrm{~min}$ at room temperature. The supernatant was replaced with $100 \mu \mathrm{l}$ permeabilization buffer following centrifugation $\left(2,000 \mathrm{x} \mathrm{g}, 10 \mathrm{~min}, 4^{\circ} \mathrm{C}\right)$ and cells were resuspended and mixed with $5 \mu \mathrm{l}(1 \mu \mathrm{g})$ monoclonal antibodies against MMP-2 and MMP-9. Following 30 min of incubation, cells were washed twice with PBS. Subsequently, cells were mixed with $100 \mu 1$ $(2.5 \mu \mathrm{g})$ secondary antibody and incubated at room temperature in the dark for $30 \mathrm{~min}$. Cells were washed twice and resuspended in PBS, prior to analysis of protein expression by flow cytometry. The mean fluorescence intensity exponent (MFI) was calculated using EXPO ${ }^{\text {Tм }} 32$ ADC software, version $1.1 \mathrm{C}$.

Assessment of VPA effects in vivo. A total of 20 female nude mice were purchased from Shanghai Laboratory Animal Center Laboratory Animal Co. Ltd (Shanghai, China) and were randomly divided into control and therapy groups $(n=10$ per group). The mice were housed in isolators under specific pathogen-free conditions with a 12/12-h light/dark cycle and an ambient temperature of $26^{\circ} \mathrm{C}$. The mice had ad libitum access to conventional nude mouse food (Shanghai Laboratory Animal Center Laboratory Animal Co. Ltd) and pH 2.5 sterile water. Exponentially growing HepG2 cells were collected, washed twice with PBS and adjusted to $1 \times 10^{7}$ cells $/ \mathrm{ml}$. Subsequently, $0.2 \mathrm{ml}$ cell suspension was subcutaneously injected into the right oxter of the mice. One week later, mice in the therapy group were administered $20 \mathrm{mg} / \mathrm{kg} / \mathrm{d}$ VPA by gastric perfusion, while control mice were administered normal 


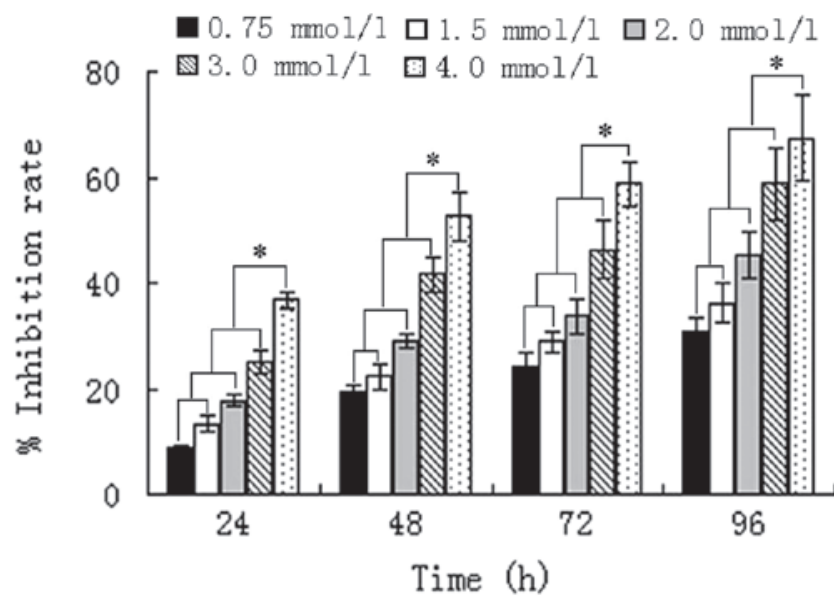

Figure 1. VPA inhibits the proliferation of HepG2 cells. HepG2 cells $\left(5 \times 10^{4}\right.$ cells $\left./ \mathrm{ml}\right)$ were incubated with VPA at $0.75,1.5,2.0,3.0$ and $4.0 \mathrm{mmol} / 1$, respectively, for the indicated time-points. Cell proliferation was determined by MTT assay. The data are based on the mean of three independent experiments, in each of which determinations were in triplicate and cell proliferation inhibition rate was calculated. Values are presented as the mean \pm standard deviation. ${ }^{*} \mathrm{P}<0.01$. VPA, sodium valproate.

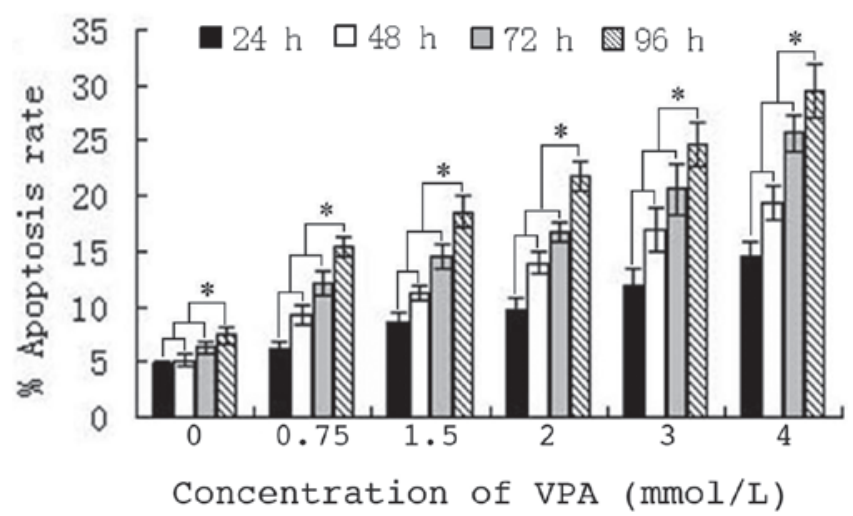

Figure 2. VPA induces apoptosis of HepG2 cells in vitro. Statistical graph of apoptosis rate of cells. Cells were seeded at $1 \times 10^{5}$ cells $/ \mathrm{ml}$ and cultured under standard conditions for the indicated time-points. Apoptosis was detected by flow cytometry using an Annexin V/propidium iodide kit. Each data point represents the mean of three separate experiments $(n=3)$. Values are presented as the mean \pm standard deviation. $\mathrm{P}<0.01$ compared with each experimental group. ${ }^{*} \mathrm{P}<0.01$. VPA, sodium valproate.

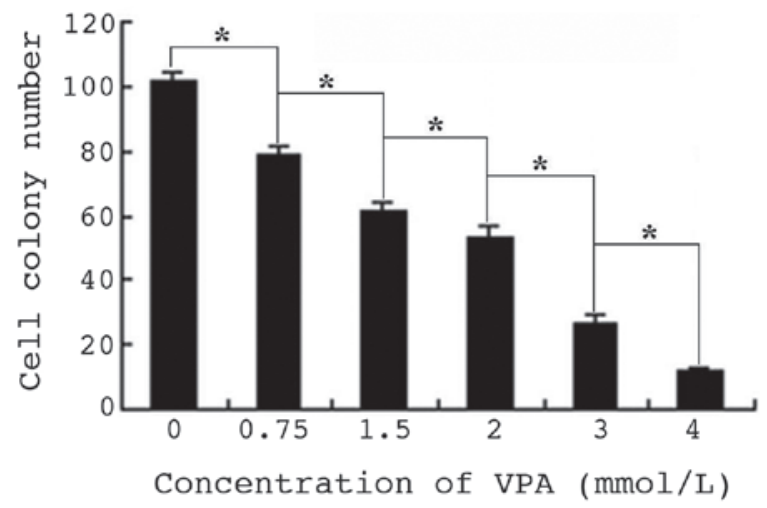

Figure 3. Effects of VPA on colony formation of HepG2 cells in vitro Colony formation of cells was measured by Giemsa staining assay following incubation of HepG2 cells with VPA for 14 days, the result was indicated as the colony number. Values are presented as the mean \pm standard deviation. ${ }^{*} \mathrm{P}<0.01$. VPA, sodium valproate. saline. Following treatment with VPA for one week, the tumor size was measured weekly and tumor volume was calculated as the formula: $V=1 / 2 x$ length $x$ width $^{2}$. Following 4 weeks of therapy, all the mice were sacrificed by cervical dislocation, the tumors were excised and weighed, and the tumor inhibition rate was calculated. Tumor inhibition rate $=$ (tumor weight of control mice - tumor weight of therapy mice)/tumor weight of control $\mathrm{x} 100 \%$. The study was approved by the ethics committee Jinan Central Hospital (Shandong University, Jinan, Shandong, China).

Statistical analysis. All experiments were performed at least in triplicate. All results are expressed as the mean \pm standard deviation. The data were analyzed with Student's unpaired $t$-test and Pearson's correlation coefficient. $\mathrm{P}<0.05$ was considered to indicate a statistically significant difference.

\section{Results}

VPA alters cell morphology and inhibits proliferation of Hep 2 cells. Control cells grew well, and were of uniform size and shape. Following exposure to VPA for $24 \mathrm{~h}$, there was no significant difference in the morphology of cells treated with 0.75 or $1.5 \mathrm{mmol} / \mathrm{l} \mathrm{VPA}$ and control cells, whereas the size and shape of 2.0-4.0 mmol/1 VPA-treated cells became varied and irregular. Following $48 \mathrm{~h}$ of incubation, the morphology of all VPA-treated cells was altered, and semi-suspended cells and apoptotic bodies were observed. In addition, condensed cell chromatin and nuclei splitting were identified. Concurrently, the proliferation of HepG2 cells was markedly inhibited from the beginning of the indicated time-points, by various concentrations of VPA $(\mathrm{P}<0.01)$. Furthermore, Pearson's correlation analysis confirmed that the inhibition of HepG2 cells by VPA was time- $(\mathrm{r}=0.403 ; \mathrm{P}=0.033)$ and dose-dependent $(\mathrm{r}=0.473$; $\mathrm{P}=0.011)$ (Fig. 1).

VPA induces apoptosis of HepG2 cells. The mechanism of proliferation inhibition of HepG2 cells by VPA was detected, following the identification of a time- and dose-dependent inhibition. The results indicated that HepG2 cell apoptosis was induced following treatment with $0.75-4.0 \mathrm{mmol} / \mathrm{l} \mathrm{VPA}$ for 24-96 h. Furthermore, the apoptotic rate increased with increasing VPA concentration and drug-exposure time. Statistical analysis confirmed that the increase in apoptosis ratio was time- $(\mathrm{r}=0.568 ; \mathrm{P}=0.004)$ and dose-dependent $(\mathrm{r}=0.786$; $\mathrm{P}<0.000$ ) (Fig. 2).

VPA inhibits colony formation of HepG2 cells. The size and number of HepG2 cell colonies decreased compared with that of control cells, following culture with VPA for 14 days (Fig. 3). The cell colony number of cells treated with $0.75,1.5$, 2.0,3.0 and 4.0 mmol/1 VPA was 79.0 $\pm 3.2,62.0 \pm 2.8,54.0 \pm 3.3$, $26.0 \pm 2.9$ and $12.0 \pm 1.1$, respectively, while that of control cells was $102 \pm 2.8(\mathrm{P}<0.01)$. Furthermore, cells treated with 3.0 and $4.0 \mathrm{mmol} / 1 \mathrm{VPA}$ exhibited notable shrinkage and the colonies formed were smaller.

VPA inhibits migration of HepG2 cells. Cell migration was determined by cell scrape assay, and observed under a microscope. Following culture for $24 \mathrm{~h}$, the mean number of 


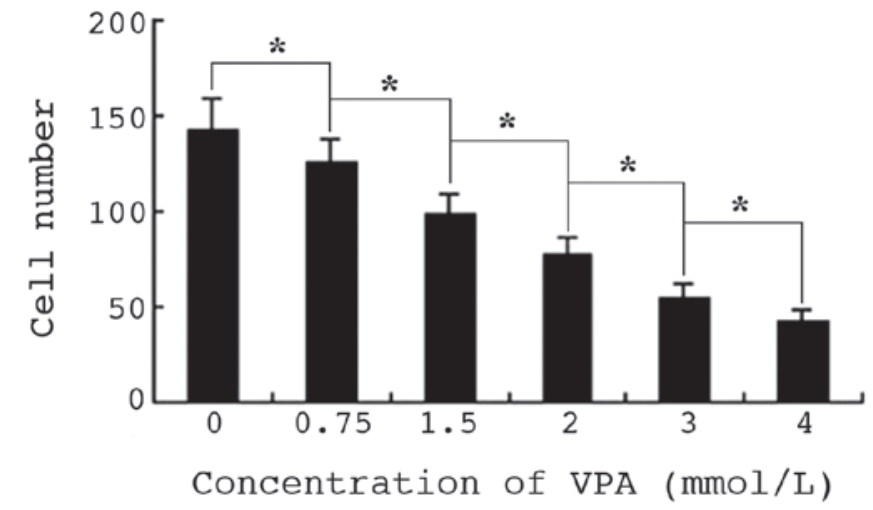

Figure 4. Effects of VPA on migration of HepG2 cells in vitro. Cell migration was observed under microscopic by scrape assay, and the results are indicated as number of cells in the scratch. Values are presented as the mean \pm standard deviation. ${ }^{*} \mathrm{P}<0.01$. VPA, sodium valproate.

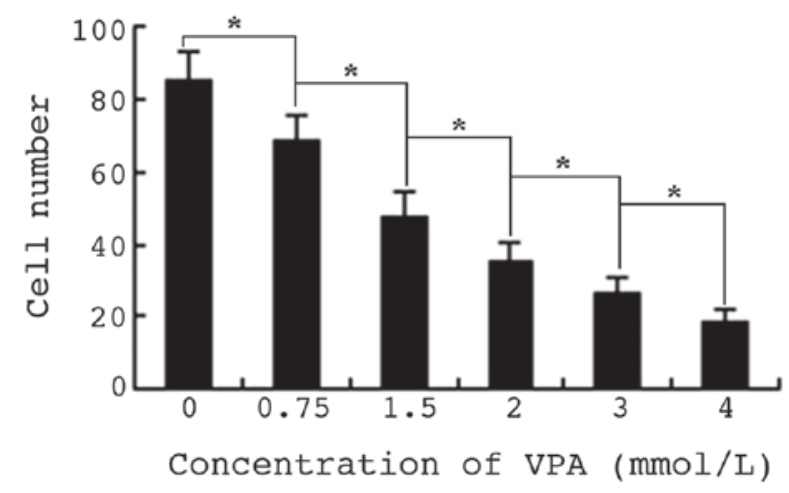

Figure 5. Effects of VPA on invasion of HepG2 cells in vitro. Cell invasion in vitro was detected by Transwell assay, and the result is indicated as the number of cells on the lower side of the polycarbonate membrane. Values are presented as the mean \pm standard deviation. ${ }^{*} \mathrm{P}<0.01$. VPA, sodium valproate.

control cells migrating to the scratch was 142 , while in the 0.75-4.0 mmol/1 VPA experimental groups, the number gradually decreased with increasing drug concentration. There were significant differences in cell migration between the 2.0-4.0 mmol/1 VPA-treated groups and cells of the 0.75 or $1.5 \mathrm{mmol} / 1$ groups $(\mathrm{P}<0.01)$. The inhibition ratios of cell migration were between 12 and $70.4 \%$, respectively, indicating a significant dose-dependent effect (Fig. 4).

VPA inhibits HepG2 cell invasion in vitro. Cell invasion in vitro was detected by Transwell assay. Cells on the lower side of the polycarbonate membrane were the invading cells, which were observed under a microscope following Wright-Giemsa staining. Following treatment with VPA, the number of invading cells was significantly reduced, compared with that of untreated cells $(\mathrm{P}<0.01)$. Furthermore, cell invasion was inhibited in a dose-dependent manner (Fig. 5).

VPA inhibits protein expression of MMP-2 and MMP-9. A total of $5 \times 10^{6} \mathrm{HepG} 2$ cells were exposed to 1.5 or $3.0 \mathrm{mmol} / 1$ VPA for $48 \mathrm{~h}$. Protein expression levels of MMP-2 and MMP-9 were analyzed by flow cytometry, and the MFI was calculated. The MFIs of MMP-2 and MMP-9 in control cells were 2.65 and 2.97, respectively. By contrast, in HepG2 cells

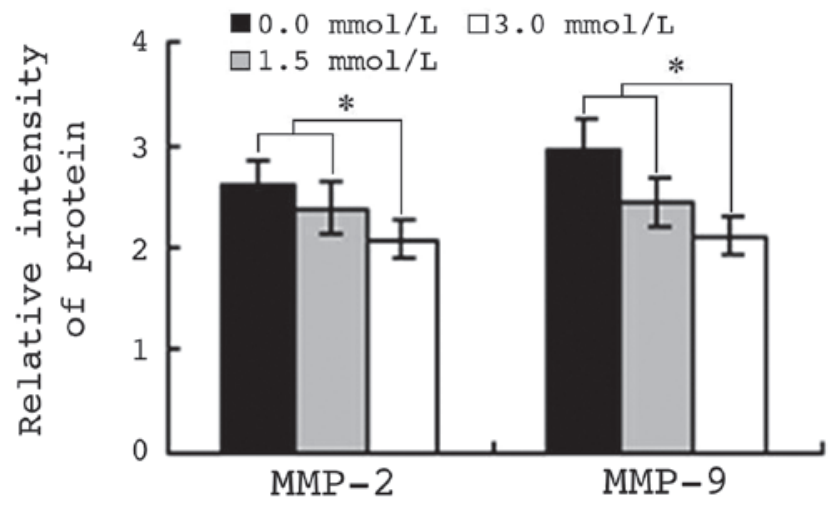

Figure 6. VPA inhibits MMP-2 and MMP-9 protein expression in HepG2 cells. The results show relative intensity of MMP-2 and MMP-9 protein expression, as determined by flow cytometry. Values are presented as the mean \pm standard deviation. ${ }^{*} \mathrm{P}<0.01$. VPA, sodium valproate.

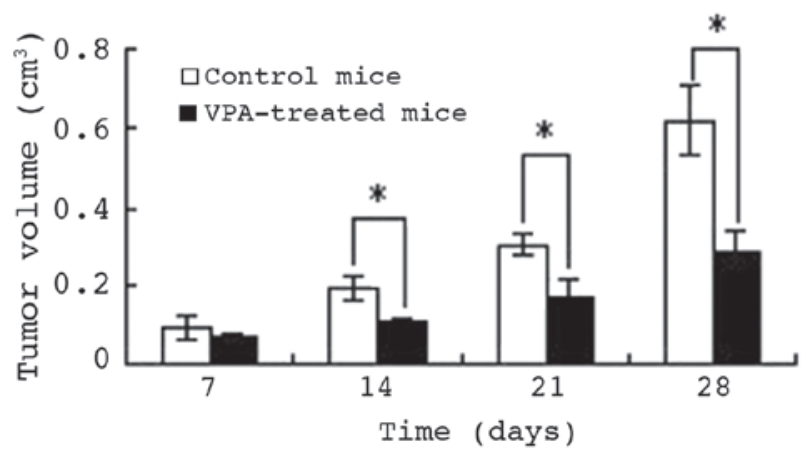

Figure 7. Antitumor effect of VPA on HepG2 cells in vivo. Tumors were induced seven days following injection with HepG2 cells, and were treated with VPA by gastric perfusion for 28 days. Tumor volume was measured on days 7, 14 and 28 . The tumor volume of control mice was greater than that of VPA-treated mice. Values are presented as the mean \pm standard deviation. ${ }^{*} \mathrm{P}<0.05$. VPA, sodium valproate.

treated with $1.5 \mathrm{mmol} / \mathrm{l} \mathrm{VPA}$ for $48 \mathrm{~h}$, the MFIs of MMP-2 and MMP-9 were 2.39 and 2.43, respectively $(\mathrm{P}<0.01)$. The MFIs of MMP-2 and MMP-9 in cells treated with $3.0 \mathrm{mmol} / 1$ VPA were 2.09 and 2.08, respectively. Pearson's correlation analysis indicated that the downregulation of MMP-2 and MMP-9 protein expression was closely correlated with the reduction in cell migration and invasion ability $(r=0.962$ and 0.950; $\mathrm{P}<0.01$ ) (Fig. 6).

VPA inhibits tumor growth in vivo. Following confirmation of the function and mechanism of VPA in HepG2 cell apoptosis, the anti-hepatocellular carcinoma effect of VPA was evaluated in vivo. Seven days following injection of HepG2 cells into nude mice, tumors were generated in 18 of the mice and VPA treatment was administered by gastric perfusion. Fig. 7 indicates that following the first week of treatment, the tumor volumes of the control and therapy mice were similar $(\mathrm{P}>0.05)$. By day 14, the tumors of VPA-treated mice were significantly smaller than those of control mice $(\mathrm{P}<0.05)$. On day 28, the tumor volume of control mice had increased to $0.62 \pm 0.10 \mathrm{~cm}^{3}$, while that of VPA therapy mice was only $0.28 \pm 0.08 \mathrm{~cm}^{3}(\mathrm{P}<0.01)$, indicating an inhibition rate of $54.84 \%$. Subsequently, the mice were sacrificed and 
the tumors weighed. The mean tumor weight of control mice was $1.49 \pm 0.38 \mathrm{~g}$, while that of therapy mice was $1.06 \pm 0.12 \mathrm{~g}$. Thus, VPA diminished the tumor weight significantly $(\mathrm{P}<0.01)$; the tumor inhibition rate was $23.82 \%$.

\section{Discussion}

In the 1950s, Cruft et al (10) reported that histones were able to bind to DNA and alter their transcriptional activities. Since then, histones have become a significant research topic (11). Histone acetylation levels are able to directly affect tumor progression, and the high expression of HDACs is closely correlated with tumorigenesis and tumor development (12). VPA is a type of short-chain fatty acid, mainly used in the treatment of hematological and nervous tumors, which has been demonstrated to be a specific HDACI (13), although its antineoplastic function has not been widely recognized. The present study focused on the malignant phenotype amelioration of HepG2 cells by VPA. In view of apoptosis as a potential mechanism for cell growth inhibition, the Annexin V/PI staining assay was used to detect apoptosis of HepG2 cells induced by VPA, following the identification of a time- and dose-dependent inhibition of HepG2 cells by VPA. The results revealed significantly enhanced apoptosis of HepG2 cells following treatment with VPA, and indicated that this effect was time- and dose-dependent.

Following culture with VPA for 14 days, the size and number of HepG2 cell colonies was decreased. Meanwhile, cell migration and invasion were also inhibited by VPA. All these effects exhibited a marked dose-dependent tendency. The MMP family is a type of zinc-dependent endopeptidase (14), and its principal function is to degrade proteins in the matrix. MMP-2 is considered to be a regulatory factor of the tumor angiogenic switch, while MMP-9 promotes angiopoiesis (15). MMPs induce the formation of small pores in the basilar membrane by degradation of the compact network structure, thereby promoting external diffusion of tumor cells through the gaps, and enhancing tumor invasion and metastasis. Furthermore, MMPs promote angiogenesis, providing more favorable conditions for tumor growth, local invasion and distant metastasis (14). The results of the present study revealed that, following culture with VPA, MMP-2 and MMP-9 protein expression levels were markedly downregulated. Pearson's correlation analysis indicated that this effect was closely correlated with the changes in cell migration and invasion ability. Therefore, downregulation of MMP-2 and MMP-9 protein expression may be one of the major mechanisms by which VPA inhibits hepatoma carcinoma cell invasion and metastasis.

Acetylation levels of the histone N-terminal may alter the state of chromatin by interfering with the affinity between histones and DNA, or disturbing the combination of transcription factor and DNA sequence (16). The regulatory role of acetylation in gene expression is similar to that of DNA genetic code, and therefore the potential role of HDACIs in tumor therapy was considered. The results of the present study indicated that regulation of histone acetylation with VPA was able to abrogate the malignant phenotype of HepG2 cells, confirming that it may be widely applied in hepatoma treatment. In addition, these results confirmed that downregulation of MMP-2 and MMP-9 protein expression may be the main mechanism by which VPA inhibits the invasion and migration of hepatocellular carcinoma cells.

\section{Acknowledgements}

The authors acknowledge the financial support of the China Postdoctoral Science Foundation, grant no. 138968.

\section{References}

1. Chen MS, Li JQ, Zhang YQ, Lu LX, Zhang WZ, Yuan YF, et al: High-dose iodized oil transcatheter arterial chemoembolization for patients with large hepatocellular carcinoma. World J Gastroenterol 8: 74-78, 2002.

2. Turner BM: Cellular memory and the histone code. Cell 111: 285-291, 2002.

3. Mottet D and Castronovo V: Histone deacetylases: Target enzymes for cancer therapy. Clin Exp Metastas 25: 183-189, 2008.

4. Weichert W, Roske A, Gekeler V, Beckers T, Ebert MP Pross M, et al: Association of patterns of class I histone deacetylase expression with patient prognosis in gastric cancer: A retrospective analysis. Lancet Oncol 9: 139-148, 2008.

5. Marks PA, Rifkind RA, Richon VM, Breslow R, Miller Y and Kelly WK: Histone deacetylases and cancer: Causes and therapies. Nat Rev Cancer 1: 194-202, 2001.

6. Pan L, Lu J and Huang B: HDAC inhibitors: A potential new category of anti-tumor agents. Cell Mol Immunol 4: 337-343, 2007.

7. Gallinari P, Marco SD, Jones P, Pallaoro M and Steinkühler C: HDACs, histone deacetylation and gene transcription: From molecular biology to cancer therapeutics. Cell Res 17: 195-211, 2007.

8. Eyal S, Yagen B, Sobol E, Altschuler Y, Shmuel M and Bialer M: The activity of antiepileptic drugs as histone deacetylase inhibitors. Epilepsia 45: 737-744, 2004.

9. Hansen MB, Nielsen SE and Berg K: Re-examination and further development of a precise and rapid dye method for measuring cell growth/cell kill. J Immunol Methods 119: 203-210, 1989.

10. Cruft HJ, Mauritzen CM and Stedman E: Abnormal properties of histones from malignant cells. Nature 174: 580-585, 1954.

11. Shi Y, Lan F, Matson C, Mulligan P, Whetstine JR, Cole PA, Casero RA and Shi Y: Histone demethylation mediated by the nuclear amine oxidase homolog LSD1. Cell 119: 941-953, 2004.

12. Seligson DB, Horvath S, Shi T, Yu H, Tze S, Grunstein M and Kurdistani SK: Global histone modification patterns predict risk of prostate cancer recurrence. Nature 435: 1262-1266, 2005.

13. Yang H, Hoshino K, Sanchez-Gonzalez B, Kantarjian H and Garcia-Manero G: Antileukemia activity of the combination of 5-aza-2'-deoxycytidine with valproic acid. Leuk Res 29: 739-748, 2005.

14. Kuwajima A, Iwashita J, Murata $\mathrm{J}$ and Abe T: The histone deacetylase inhibitor butyrate inhibits melanoma cell invasion of Matrigel. Anticancer Res 27: 4163-4169, 2007.

15. Sun LC, Luo J, Mackey LV, Fuselier JA and Coy DH: A conjugate of camptothecin and a somatostatin analog against prostate cancer cell invasion via a possible signaling pathway involving PI3K/Akt, alphaVbeta3/alphaVbeta5 and MMP-2/-9. Cancer Lett 246: 157-166, 2007.

16. Esteller M: Cancer epigenomics: DNA methylomes and histone-modification maps. Nat Rev Genet 8: 286-298, 2007. 